\title{
VARIABLE EXPONENT LEBESGUE SPACES \\ ON METRIC SPACES: \\ THE HARDY-LITTLEWOOD MAXIMAL OPERATOR
}

\author{
PETTERI HARJULEHTO, PETER HÄSTÖ AND MIKKO PERE
}

\begin{abstract}
In this article we introduce variable exponent Lebesgue spaces on metric measure spaces and consider a central tool in geometric analysis: the HardyLittlewood maximal operator. We show that the maximal operator is bounded provided the variable exponent satisfies a log-Hölder type estimate. This condition is known to be essentially sharp in real Euclidean space, however, we show that this is not so in metric spaces.
\end{abstract}

\section{INTRODUCTION}

Lebesgue and Sobolev spaces defined on metric spaces are to-date well understood, see for instance [12]. These are function spaces in which we replace the Euclidean space from classical analysis by a metric space equipped with a measure (satisfying some conditions). Such spaces are natural abstractions in many situations - consider for instance a plate joined to a block, or a rod joined to a plate (Figure 1). Neither of these domains can be adequately analyzed in Euclidean space, since the joined plate or rod has measure zero.

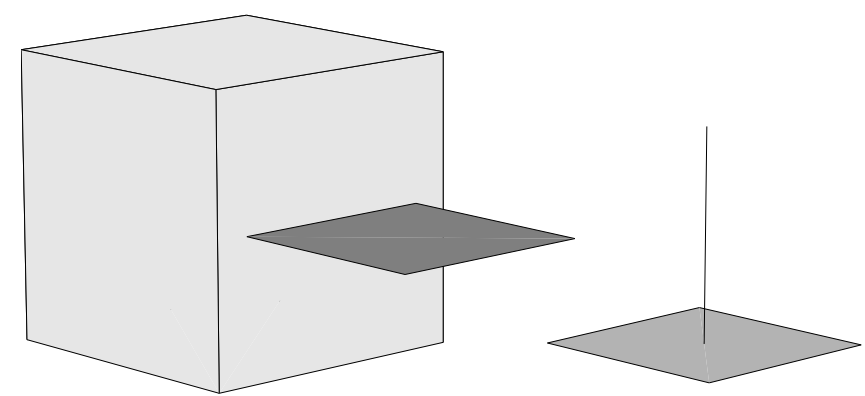

Figure 1. Some metric spaces

In classical Lebesgue and Sobolev theory many properties depend crucially on the dimension of the underlying Euclidean space. Examples include the boundedness of convolution with a Riesz kernel and the Sobolev embedding. In general, metric measure spaces do not have a dimension which could reasonably take the place of the Euclidean dimension in these cases. This problem has been "dealt with" by considering spaces with a doubling measure (see $[8,9,16]$, for examples). However,

Date: October 6, 2003.

2000 Mathematics Subject Classification. 42B25, 42B33.

Key words and phrases. Variable exponent, Lebesgue space, metric measure space, maximal operator. 
this really gives only a lower dimension, i.e. $\mu(B(x, r)) \geq C r^{Q}$. Moreover, this dimension is the same in every part of the space, which does not correspond to the geometry of the domain. To tackle this problem we propose that analysis on metric measure spaces should be done in Lebesgue spaces where the exponent is allowed to vary, to reflect the non-homogeneity of the underlying space. Then perhaps a local uniformness condition of the kind $\mu(B(x, r)) \approx r^{q(x)}$ will suffice for many properties. Note that this condition is satisfied in the spaces shown in Figure 1.

Variable exponent Lebesgue spaces on Euclidean spaces have attracted a steadily increasing interest over the last couple of years, but the variable exponent framework has not yet been applied to the metric measure space setting. Variable exponent spaces have been independently discovered by several investigators [7, 14, 20, 21]. These investigations were motivated by differential equations with non-standard coercivity conditions, arising for instance from modeling certain fluids (e.g, [1, 6, 19]). For some of the latest advances see $[4,5,10,13]$. Obviously, we also expect that the greater versatility of variable exponent spaces will usefully carry over to the metric space setting.

In this article we initiate the investigation of variable exponent Lebesgue spaces on metric spaces by considering the Hardy-Littlewood maximal operator $\mathcal{M}$. It has proved to be a very useful tool in geometric analysis both in $\mathbb{R}^{n}$ and in metric measure spaces. One central question is whether $\mathcal{M}$ maps $L^{p}$ to $L^{p}$. In metric spaces with a doubling measure everything works as in the classical case, i.e. $L^{p}$ maps to itself provided $p>1$, [12]. In variable exponent Lebesgue spaces on $\mathbb{R}^{n}$ the situation is a bit more precarious: $L^{p(\cdot)}$ maps to $L^{p(\cdot)}$ only when $p(\cdot)$ is sufficiently regular. Due to the efforts of L. Pick \& M. Růžička [18], L. Diening [3], A. Nekvinda [17], and D. Cruz-Uribe, A. Fiorenze \& C. Neugebauer [2], the essentially sharp condition on $p(\cdot)$ is known.

We will show in this paper that the condition from the Euclidean setting is sufficient but not necessary in metric measure spaces (Theorem 4.3 and Example 4.5, respectively). For the first of these results we adapt a method of L. Diening's from [3]. In Section 5 we show that a so-called weak type estimate of the maximal operator holds irrespective of the variation of the exponent. This result and its proof are similar to their analogues in Euclidean spaces, [2, Section 4]. D. Cruz-Uribe, A. Fiorenze and C. Neugebauer [2] have also given the correct condition for when the maximal operator is bounded on Lebesgue spaces defined on the whole of $\mathbb{R}^{n}$. Unfortunately, e are not able to generalize this global result. We conclude by giving a weak type estimate of the maximal operator (adapting a method from [2]) and use it to show that almost every point is a Lebesgue point of a function in $L_{l o c}^{p(\cdot)}(X)$.

\section{Metric measure spaces}

By a metric measure space we mean a triple $(X, d, \mu)$, where $X$ is a set, $d$ is a metric on $X$ and $\mu$ is a non-negative Borel regular outer measure on $X$ which is finite in every bounded set. For simplicity, we often write $X$ instead of $(X, d, \mu)$. For $x \in X$ and $r>0$ we denote by $B(x, r)$ the open ball centered at $x$ with radius $r$. We use the convention that $C$ denotes a constant whose value can change even between different occurrences on the same line.

A metric measure space $X$ or a measure $\mu$ is said to be doubling if there is a constant $C \geq 1$ such that

$$
\mu(B(x, 2 r)) \leq C \mu(B(x, r))
$$


for every open ball $B(x, r) \subset X$. The constant $C$ in $(2.1)$ is called the doubling constant of $\mu$. By the doubling property, if $B(y, R)$ is an open ball in $X, x \in B(y, R)$ and $0<r \leq R<\infty$, then

$$
\frac{\mu(B(x, r))}{\mu(B(y, R))} \geq C_{Q}\left(\frac{r}{R}\right)^{Q}
$$

for some $C_{Q}$ and $Q$ depending only on the doubling constant. For example, in $\mathbb{R}^{n}$ with the Lebesgue measure (2.2) holds with $Q$ equal to the dimension $n$.

We say that the measure $\mu$ is lower Ahlfors $Q$-regular if there exists a constant $C>0$ such that $\mu(B) \geq C \operatorname{diam}(B)^{Q}$ for every ball $B \subset X$ with $\operatorname{diam} B \leq 1$. We say that $\mu$ is upper Ahlfors q-regular if there exists a constant $C>0$ such that $\mu(B) \leq C \operatorname{diam}(B)^{q}$ for every ball $B \subset X$ with $\operatorname{diam} B \leq 1$. The measure $\mu$ is Ahlfors $q$-regular if it is upper and lower Ahlfors $q$-regular, i.e. if $\mu(B) \approx \operatorname{diam}(B)^{q}$ for every ball $B \subset X$ with $\operatorname{diam} B \leq \operatorname{diam} X$.

2.3. Lemma. Let $X$ be a bounded metric measure space, i.e. $\mu(X)<\infty$ and $\operatorname{diam}(X)<$ $\infty$. If $\mu$ is a doubling measure, then it is lower Ahlfors $Q$-regular.

Proof. By property (2.2) we obtain for every $x \in X$ and $0<r<\operatorname{diam}(X)$

$$
\mu(B(x, r)) \geq C_{Q} \frac{\mu(X)}{\operatorname{diam}(X)^{Q}} r^{Q}=C_{Q, X} r^{Q} .
$$

\section{VARiable EXPonent Lebesgue spaces}

We call a measurable function $p: X \rightarrow[1, \infty)$ a variable exponent. For $A \subset X$ we define $p_{A}^{+}=\operatorname{ess}_{\sup _{x \in A}} p(x)$ and $p_{A}^{-}=\operatorname{essinf}_{x \in A} p(x)$; we use the abbreviations $p^{+}=p_{X}^{+}$and $p^{-}=p_{X}^{-}$. For a $\mu$-measurable function $u: X \rightarrow \mathbb{R}$ we define the modular

and the norm

$$
\varrho_{p(\cdot)}(u)=\int_{X}|u(y)|^{p(y)} d \mu(y)
$$

$$
\|u\|_{p(\cdot)}=\inf \left\{\lambda>0: \varrho_{p(\cdot)}(u / \lambda) \leq 1\right\} .
$$

Sometimes we use the notation $\|u\|_{p(\cdot), X}$ when we also want to indicate in what metric space the norm is taken. The variable exponent Lebesgue spaces on $X, L^{p(\cdot)}(X, d, \mu)$, consists of those $\mu$-measurable functions $u: X \rightarrow \mathbb{R}$ for which there exists $\lambda>0$ such that $\varrho_{p(\cdot)}(\lambda u)<\infty$. This space is an Orlicz-Museliak space (at least in Euclidean space), cf. [15].

It is easy to see that $\|\cdot\|_{p(\cdot)}$ is a norm. As in [14] we see that if $\|f\|_{p(\cdot)} \leq 1$ then $\varrho_{p(\cdot)}(f) \leq\|f\|_{p(\cdot)}$. Moreover, if $p^{+}<\infty$, then $\varrho_{p(\cdot)}\left(f_{i}\right) \rightarrow 0$ if and only if $\left\|f_{n}\right\|_{p(\cdot)} \rightarrow 0$. Hölder's inequality (i.e. $\left.\|f g\|_{1} \leq C\|f\|_{p(\cdot)}\|g\|_{p^{\prime}(\cdot)}\right)$ holds also in variable exponent Lebesgue spaces, the proof being as in the Euclidean setting, [14, Theorem $2.1]$.

3.1. Lemma. The space $L^{p(\cdot)}(X)$ is a Banach space.

Proof. Let $\left(f_{i}\right)$ be a Cauchy sequence in $L^{p(\cdot)}(X)$. Then there is a subsequence, denoted by $\left(f_{i}\right)$, such that

$$
\left\|f_{i+1}-f_{i}\right\|_{p(\cdot)}<2^{-i}
$$

We set

$$
g_{k}=\sum_{i=1}^{k}\left|f_{i+1}-f_{i}\right| \text { and } g=\sum_{i=1}^{\infty}\left|f_{i+1}-f_{i}\right| .
$$


By (3.2) and the triangle inequality, we obtain $\left\|g_{k}\right\|_{p(\cdot)}<1$ for every $k$. Fatou's Lemma applied to $\left(g_{k}^{p(\cdot)}\right)$ gives

$$
\int_{X} \liminf g_{k}(x)^{p(x)} d \mu(x) \leq \liminf \int_{X} g_{k}(x)^{p(x)} d \mu(x) \leq\left\|g_{k}\right\|_{p(\cdot)}<1 .
$$

In particular $g(x)<\infty$ almost everywhere, so that

$$
f=f_{1}+\sum_{i=1}^{\infty}\left(f_{i+1}-f_{i}\right)
$$

exists almost everywhere, and $f(x)=\lim f_{i}(x)$ almost everywhere. Now we need to prove that $f$ is the $L^{p(\cdot)}$ limit of $\left(f_{i}\right)$. Let $\varepsilon>0$. There exists $N$ such that $\left\|f_{i}-f_{j}\right\|_{p(\cdot)}<\varepsilon$ for $i, j>N$. For every $m>N$, Fatou's Lemma implies that

$$
\int_{X}\left|f-f_{m}\right|^{p(\cdot)} d \mu \leq \liminf _{i \rightarrow \infty} \int_{X}\left|f_{i}-f_{m}\right|^{p(\cdot)} d \mu \leq \liminf _{i \rightarrow \infty}\left\|f_{i}-f_{m}\right\|_{p(\cdot)} \leq \varepsilon
$$

and hence $f \in L^{p(\cdot)}(X)$ and $\left\|f-f_{m}\right\|_{p(\cdot)} \rightarrow 0$ as $m \rightarrow \infty$.

In the next theorem we use the method of Kováčik and Rákosník [14, Theorem 2.11] to show that continuous functions are dense in variable exponent Lebesgue space.

3.3. Theorem. Let $X$ be a locally compact doubling space and let $p^{+}<\infty$. Then continuous functions with compact support are dense in $L^{p(\cdot)}(X)$.

Proof. Let $f \in L^{p(\cdot)}(X)$ and define

$$
f_{n}(x)= \begin{cases}f(x), & \text { if }|f(x)| \leq n \text { and } x \in B(0, n) \\ n \operatorname{sign} f(x), & \text { if }|f(x)|>n \text { and } x \in B(0, n), \\ 0, & \text { elsewhere }\end{cases}
$$

Then each $f_{n}$ is bounded and has a bounded support. By the Lebesgue Dominated Convergence Theorem $\rho_{p(\cdot)}\left(f-f_{n}\right) \rightarrow 0$ as $n \rightarrow \infty$, and therefore also $\left\|f_{n}-f\right\|_{p(\cdot)} \rightarrow 0$. This shows that the set of bounded functions with bounded support is dense in $L^{p(\cdot)}$.

Let $\epsilon>0$ and choose a bounded function $g \in L^{p(\cdot)}(X)$ with bounded support such that

$$
\|f-g\|_{p(\cdot)}<\epsilon .
$$

By Luzin's theorem [22, Theorem 11.36] there exists a continuous function $h$ with compact support in $X$ and an open set $U$ such that

$$
\mu(U)<\min \left\{1,\left(\frac{\epsilon}{2\|g\|_{\infty}}\right)^{p^{+}}\right\}
$$

$g(x)=h(x)$ in $X \backslash U$, and $\sup |h(x)|=\sup _{X \backslash U}|g(x)| \leq\|g\|_{\infty}$. Hence,

$$
\rho_{p(\cdot)}((g-h) / \epsilon) \leq \max \left\{1,\left(2\|g\|_{\infty} / \epsilon\right)^{p^{+}}\right\} \mu(U) \leq 1,
$$

which implies $\|g-h\|_{p(\cdot)} \leq \epsilon$. Together with (3.4) this gives

$$
\|f-h\|_{p(\cdot)} \leq 2 \epsilon .
$$


The following condition has emerged as the right one to guarantee regularity of variable exponent Lebesgue spaces. We say that $p$ is $\log$-Hölder continuous if

$$
|p(x)-p(y)| \leq \frac{C}{-\log d(x, y)},
$$

when $d(x, y) \leq 1 / 2$. This condition has also been called Dini-Lipschitz, weakLipschitz and 0-Hölder. Since it is the limiting case of $\alpha$-Hölder continuity, we think that the term log-Hölder is the most descriptive one. The following lemma illustrates the geometrical significance of log-Hölder continuous exponents. It corresponds to Lemma 3.2 of [3].

3.6. Lemma. Assume that $p^{+}<\infty$ and define two conditions:

(1) $p$ is log-Hölder continuous,

(2) for all balls $B \subset X$ we have $\mu(B)^{p_{B}^{-}-p_{B}^{+}} \leq C$.

If $\mu$ is lower Ahlfors $Q$-regular, then (1) implies (2). If $\mu$ is upper Ahlfors q-regular, then (2) implies (1).

Proof. Suppose first that $\mu$ is lower Ahlfors $Q$-regular and that (1) holds. Since $p_{B}^{-}-p_{B}^{+} \leq 0$, it suffices to check (2) for balls $B$ with radius $r$ less than $\frac{1}{2}$. By (3.5) we obtain

$$
\mu(B)^{p_{B}^{-}-p_{B}^{+}} \leq\left(C r^{Q}\right)^{p_{B}^{-}-p_{B}^{+}} \leq(C r)^{\frac{-Q C}{\log (1 / 2 r)}} \leq C .
$$

Suppose then that $\mu$ is upper Ahlfors $q$-regular and that (2) holds. Since $p^{+}<\infty$, we need only prove (3.5) for $d(x, y)$ less than some constant. We choose this constant so that $\mu(B(x, d(x, y)))<1$ for all pairs $x, y$ that we consider. For these $x$ and $y$ and $B=B(x, d(x, y))$ we have

$$
|p(x)-p(y)| \leq C\left(p_{B}^{-}-p_{B}^{+}\right) \frac{\log \mu(B)}{-\log d(x, y)} \leq \frac{C}{-\log d(x, y)} .
$$

\section{A strong type estimate}

For every locally integrable function on $X$ we set

$$
\mathcal{M}_{B(x, r)} f=f_{B(x, r)}|f(y)| d \mu(y)
$$

and

$$
\mathcal{M} f(x)=\sup _{r>0} \mathcal{M}_{B(x, r)} f .
$$

The operator $\mathcal{M}$ is called the Hardy-Littlewood maximal operator. If $X$ is doubling, then for every $t>0$ and $f \in L^{1}(X)$ we have the weak type estimate

$$
\mu(\{x \in X: \mathcal{M} f(x)>t)\}) \leq \frac{C_{1}}{t} \int_{X}|f| d \mu
$$

and for every $f \in L^{q}(X), 1<q \leq \infty$, the strong type estimate

$$
\|\mathcal{M} f\|_{q, X} \leq C_{q}\|f\|_{q, X} .
$$

The constants $C_{1}$ and $C_{q}$ depend only on $q$ and the doubling constant. Proof for these results can be found for example in [12].

Our first goal is to generalize the latter result to variable exponent Lebesgue spaces, adapting the method of L. Diening's from [3]. The following lemma is a streamlined version of [3, Lemma 3.3]. 
4.2. Lemma. Suppose that $X$ is a bounded doubling space and that $p$ is log-Hölder continuous with $p^{+}<\infty$. Let $f \in L^{p(\cdot)}(X, \mu)$ be such that $(1+\mu(X))\|f\|_{p(\cdot)} \leq 1$. Then for every $x \in X$ we have

$$
[\mathcal{M} f(x)]^{p(x)} \leq C\left(\mathcal{M}\left(f^{p}\right)(x)+1\right) .
$$

Proof. Fix $x \in X$ and let $B$ be a ball centered at $x$. By [14, Theorem 2.8], we have $\|f\|_{p_{B}^{-}} \leq(1+\mu(X))\|f\|_{p(\cdot)} \leq 1$. Using Hölder's inequality, $\|f\|_{p_{B}^{-}} \leq 1$ and Lemmata 2.3 and 3.6 we find that

$$
\begin{aligned}
\left(\mathcal{M}_{B} f\right)^{p(x)} & \leq\left(f_{B}|f(y)|^{p_{B}^{-}} d \mu(y)\right)^{p(x) / p_{B}^{-}}=\mu(B)^{-p(x) / p_{B}^{-}}\|f\|_{p_{B}^{-}}^{p(x)} \\
& \leq \mu(B)^{-p(x) / p_{B}^{-}}\|f(y)\|_{p_{B}^{-}}^{p_{B}^{-}}=\mu(B)^{\left(p_{B}^{-}-p(x)\right) / p_{B}^{-}} \int_{B}|f(y)|^{p_{B}^{-}} d \mu(y) \\
& \leq C f_{B}|f(y)|^{p(y)}+1 d \mu(y) \leq C\left(\mathcal{M}_{B}\left(f^{p}\right)+1\right) .
\end{aligned}
$$

Since the constant does not depend on the ball $B$, the result follows by taking the supremum over $B$.

We are now ready to prove the main result, which corresponds to Theorem 3.5, $[3]$.

4.3. Theorem. Let $X$ be a bounded doubling space. Suppose that $p$ is log-Hölder continuous with $1<p^{-} \leq p^{+}<\infty$. Then

$$
\|\mathcal{M} f\|_{p(\cdot)} \leq C\|f\|_{p(\cdot)} \text {. }
$$

Proof. Define $q(x)=p(x) / p^{-}$for $x \in X$. Since (4.4) is homogeneous, it suffices to assume that $\|f\|_{p(\cdot)} \leq 1$ and prove that $\|\mathcal{M} f\|_{p(\cdot)} \leq C$. We may assume additionally that $(1+\mu(X))\|f\|_{q(\cdot)} \leq 1$. Then $f$ satisfies the assumptions of Lemma 4.2 (with $p$ replaced by $q)$, and so

$$
[\mathcal{M} f(x)]^{q(x)} \leq C\left(\mathcal{M}\left(f^{q}\right)(x)+1\right)
$$

for every $x \in X$. Since $p^{-}>1, \mathcal{M}: L^{p^{-}} \rightarrow L^{p^{-}}$is a bounded operator, and we get

$$
\begin{aligned}
\rho_{p(\cdot)}(\mathcal{M} f)=\left\|(\mathcal{M} f)^{q(\cdot)}\right\|_{p^{-}}^{p^{-}} & \leq C\left(\left\|\mathcal{M}\left(f^{q}\right)\right\|_{p^{-}}+\|1\|_{p^{-}}\right)^{p^{-}} \\
& \leq C\left(\left\|f^{q}\right\|_{p^{-}}+\mu(X)^{1 / p^{-}}\right)^{p^{-}} \leq C .
\end{aligned}
$$

Since $p^{+}<\infty$, this implies (by [7, Theorem 1.3]) that $\|\mathcal{M} f\|_{p(\cdot)} \leq C$, which proves the claim.

An example by L. Pick and M. Růžička [18] showed that log-Hölder continuity of the exponent is essentially the optimal condition for when the maximal operator is bounded on variable exponent Lebesgue spaces defined on Euclidean spaces. The next example features a metric space of the kind considered in the introduction. It shows that in metric spaces the maximal function can be bounded even though the variable exponent is not log-Hölder continuous. In particular, this example implies that there is still work to be done on this question in metric spaces. 
4.5. Example. Let $X_{1}=\left\{(x, 0) \in \mathbb{R}^{2}: 0 \leq x<1 / 4\right\}$ and $X_{2}=\{(x, y) \in$ $B(0,1 / 2): x<0\}$ and define $(X, \mu)=\left(X_{1}, m_{1}\right) \cup\left(X_{2}, m_{2}\right)$, where $m_{i}$ denotes the $i$-dimension Lebesgue measure. The space $X$ is the leftmost domain in Figure 2 . We set the exponent $p$ equal to $s$ on $X_{1}$ and to $t$ on $X_{2}(s, t>1)$. Then $\mathcal{M}$ is a bounded operator from $L^{p(\cdot)}(X, \mu)$ onto itself if and only if $s \geq t$ and either $t \geq 2$ or $s<t /(2-t)$.
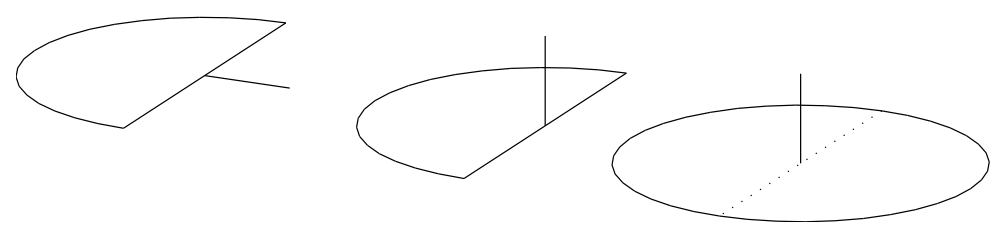

Figure 2. The space $X$ and some variants

4.6. Remark. The space $X$ in the previous example is essentially the same as the right-hand-side space in Figure 1, since $X$ is mapped by a bilipshcitz mapping to the space in the middle of Figure 2 and can then be extended to the third space in Figure 2 by reflexion. In particular, the maximal operator is bounded for exactly the same exponents in all these cases.

Let us prove the claims of the example. Take $f \in L^{p(\cdot)}(X, \mu)$. For the ball $B \subset X$ we have

$$
\mathcal{M}_{B} f=\frac{1}{m_{1}\left(B \cap X_{1}\right)+m_{2}\left(B \cap X_{2}\right)}\left(\int_{B \cap X_{1}}|f(y)| d m_{1}(y)+\int_{B \cap X_{2}}|f(y)| d m_{2}(y)\right) .
$$

Suppose first that $s \geq t$ and either $t \geq 2$ or $s<t /(2-t)$. If we denote $f_{1}=f \chi_{X_{1}}$ and $f_{2}=f \chi_{X_{2}}\left(\chi_{S}\right.$ denotes the characteristic function of the set $\left.S\right)$, then we see that

$$
\|\mathcal{M} f\|_{p(\cdot)} \leq\left\|\mathcal{M} f_{1}\right\|_{s, X_{1}}+\left\|\mathcal{M} f_{2}\right\|_{s, X_{1}}+\|\mathcal{M} f\|_{t, X_{2}} .
$$

Since $f \in L^{p(\cdot)}(X) \subset L^{t}(X)$, classical Lebesgue theory in metric spaces tells us that the third term in the last expression is bounded by $C\|f\|_{p(\cdot), X}$. Since the first term lives only in $X_{1}$ and $f_{1} \in L^{s}(X)$, we get an upper bound for it in terms of $\left\|f_{1}\right\|_{s, X_{1}}$. Therefore we have shown that

$$
\|\mathcal{M} f\|_{p(\cdot)} \leq C\left(\left\|f_{1}\right\|_{s, X_{1}}+\|f\|_{p(\cdot), X}\right)+\left\|\mathcal{M} f_{2}\right\|_{s, X_{1}}
$$

and all that remains is to show is that $\left\|\mathcal{M} f_{2}\right\|_{s, X_{1}} \leq C\left\|f_{2}\right\|_{t, X_{2}}$.

The idea of the proof is that the only really important part of the norm is the origin and its surroundings. Therefore we can make estimates when calculating the norms that have an effect only far from the origin, without this having a large effect on the result.

In what follows we will denote by $B(r)$ a ball centered at the origin with radius $r>0$. For a ball $B$ centered on $X_{1}$ and intersecting $X_{2}$, let $B^{\prime}$ be the ball whose diameter is $B \cap\{(0, y): y \in \mathbb{R}\}$. Then for $x \in X_{1}$ and a ball $B$ centered at $x$ we have

$$
\begin{aligned}
\mathcal{M}_{B} f_{2} & =f_{B}\left|f_{2}\right| d \mu \leq \frac{1}{\mu(B)} \int_{B^{\prime}}\left|f_{2}\right| d \mu \leq \frac{1}{\mu\left(B \cap X_{1}\right)} \int_{B^{\prime} \cap X_{2}}\left|f_{2}\right| d m_{2} \\
& \leq \frac{1}{\min \{|x|+r, 1 / 4\}} \int_{B^{\prime} \cap X_{2}}\left|f_{2}\right| d m_{2},
\end{aligned}
$$


where $r$ is the radius of $B^{\prime}$. Therefore

$$
\mathcal{M} f_{2}(x) \leq \frac{1}{\min \{|x|+r(x), 1 / 4\}} \int_{B(r(x)) \cap X_{2}}\left|f_{2}\right| d m_{2}
$$

for some suitable function $r: X_{1} \rightarrow[0,1 / 2)$. For those $x \in X_{1}$ with $|x|+r(x)>1 / 4$ this means that

$$
\mathcal{M} f_{2}(x) \leq 4 \int_{X_{2}}\left|f_{2}\right| d m_{2} \leq C\left\|f_{2}\right\|_{t, X_{2}},
$$

so we need not worry about these points. For the other points we calculate

$$
\left\|\mathcal{M} f_{2}\right\|_{s, X_{1}} \leq\left(\int_{0}^{1 / 4}\left(\frac{1}{|x|+r(x)} \int_{B(r(x)) \cap X_{2}}\left|f_{2}\right| d m_{2}\right)^{s} d x\right)^{1 / s} .
$$

By Hölder's inequality we have

$$
\int_{B(r) \cap X_{2}}\left|f_{2}\right| d m_{2} \leq\left\|f_{2}\right\|_{t, B(r) \cap X_{2}}\|1\|_{t^{\prime}, B(r) \cap X_{2}} \leq C r^{2(1-1 / t)}\left\|f_{2}\right\|_{t, X_{2}} .
$$

Using this in (4.7) gives

$$
\begin{aligned}
\left\|\mathcal{M} f_{2}\right\|_{s, X_{1}} & \leq\left(\int_{0}^{1 / 4}\left(\frac{C r(x)^{2(1-1 / t)}}{|x|+r(x)}\left\|f_{2}\right\|_{t, X_{2}}\right)^{s} d x\right)^{1 / s} \\
& =C\left(\int_{0}^{1 / 4}\left(\frac{r(x)^{2(1-1 / t)}}{|x|+r(x)}\right)^{s} d x\right)^{1 / s}\left\|f_{2}\right\|_{t, X_{2}} .
\end{aligned}
$$

By considering the partial derivative with respect to $r$, we see that

$$
\frac{r^{a}}{|x|+r} \leq \begin{cases}\frac{a^{a}}{(1-a)^{1-a}} x^{1-2 / t} & \text { if } a<1 \\ \frac{1}{1+x} & \text { if } a \geq 1\end{cases}
$$

where $a=2(1-1 / t)$. Using this in our previous estimate gives $\left\|\mathcal{M} f_{2}\right\|_{s, X_{1}} \leq$ $C\left\|f_{2}\right\|_{t, X_{2}}$ if either $a \geq 1$ (that is $t \geq 2$ ), or $a<1$ and

$$
\int_{0}^{1 / 4} x^{(1-2 / t) s} d x<\infty .
$$

This integral is finite provided $(1-2 / t) s>-1$, i.e. $s<t /(2-t)$, which concludes the proof of sufficiency.

If $t>s$, it is easy to see that the maximal operator is not bounded (consider the function defined as $|x|^{-2 /(s+t)}$ on $X_{1}$ and 0 on $\left.X_{2}\right)$. For $t<2$ we will construct a function $g \in L^{p(\cdot)}(X)$ for which $\mathcal{M} g \notin L^{t /(2-t)}\left(X_{1}\right)$. Since $L^{s}\left(X_{1}\right) \subset L^{t /(2-t)}\left(X_{1}\right)$ for $s>t /(2-t)$, this will conclude the proof. We set $s=t /(2-t)$.

If we analyze the proof of sufficiency above, we see that we need to choose a function with $g^{t} \in L^{1} \backslash L \log L$ for there to be any chance of our example working. It turns out that $g(x)=(|x| \log (1 /|x|))^{-2 / t}$ for $x \in X_{2}$ and $\left.g\right|_{X_{1}}=0$ does the trick. For we have

$$
\|g\|_{t, X_{2}}^{t}=\int_{0}^{1 / 2} r(r \log (1 / r))^{-2} d r=\int_{0}^{1 / 2} r^{-1} \log ^{-2}(1 / r) d r=\frac{1}{\log 2}
$$


so that $g \in L^{t}\left(X_{2}\right)$. For $x \in X_{1}$ we find that

$$
\begin{aligned}
\mathcal{M} g(x) & \geq \int_{B(x, 2|x|)}|g(y)| d \mu(y) \geq \frac{1}{3|x|+\pi(2|x|)^{2}} \int_{B(0,|x|)} g(y) d m_{2}(y) \\
& =\frac{1}{3+4 \pi|x|} \int_{0}^{|x|} r g(r) d r \geq \frac{1}{3+\pi} f_{0}^{|x|} r^{1-2 / t} \log ^{-2 / t}\left(\frac{1}{r}\right) d r .
\end{aligned}
$$

Since $r \mapsto r^{1-2 / t} \log ^{-2 / t}(1 / r)$ is convex, we find, by Jensen's inequality, that

$$
\int_{0}^{2 y} r^{1-2 / t} \log ^{-2 / t}\left(\frac{1}{r}\right) d r \geq y^{1-2 / t} \log ^{-2 / t}\left(\frac{1}{y}\right) \text {. }
$$

Using this in the previous estimate gives

$$
\begin{aligned}
\|\mathcal{M} g\|_{s, X_{1}}^{s} & =\int_{0}^{1 / 4}(\mathcal{M} g(x))^{s} d x \geq \frac{1}{(3+\pi)^{s}} \int_{0}^{1 / 4}\left((x / 2)^{1-2 / t} \log ^{-2 / t}\left(\frac{2}{x}\right)\right)^{s} d x \\
& \geq \frac{2}{(3+\pi)^{s}} \int_{0}^{1 / 8} y^{-1} \log ^{-2 /(2-t)}\left(\frac{1}{y}\right) d y .
\end{aligned}
$$

This last integral is finite if and only if $2 /(2-t)>1$, i.e. $t<1$. But since $t>1$ by assumption, this means that $\mathcal{M} g \notin L^{s}\left(X_{1}\right)$, which was to be shown.

\section{A Weak type estimate and Lebesgue points}

In this section we derive a weak type inequality of the norm of the maximal operator. This result is obtained by adapting the method used by Cruz-Uribe, Fiorenze and Neugebauer in Section 4, [2]. Their result is stated assuming that the inverse of the variable exponent belongs to the reverse Hölder class. We will assume instead that $p^{+}<\infty$. This stronger assumption is motivated by the fact that almost nothing is known of the properties of variable exponent Lebesgue or Sobolev spaces when $p^{+}=\infty$. Also our assumption allows us to simplify and shorten their proof.

The next lemma is an adaptation of [2, Lemma 4.1]

5.1. Lemma. Let $X$ be a doubling space and assume that $p^{+}<\infty$. Let also $f \in$ $L^{p(\cdot)}(X)$ and $t>0$. Suppose that $B$ is a ball such that

$$
\int_{B} \frac{|f(y)|}{t} d \mu(y)>\mu(B)
$$

Then there exists a constant $C$ depending only on $p$ such that

$$
\mu(B) \leq C \int_{B}\left(\frac{|f(y)|}{t}\right)^{p(y)} d \mu(y) .
$$

Proof. We choose a sequence of simple functions $\left\{s_{n}\right\}$ with $s_{n} \geq p_{B}^{-}$such that the sequence $\left\{s_{n}(x)\right\}$ increases monotonically to $p(x)$ on $B$. Then for each $n$,

$$
s_{n}(x)=\sum_{j=1}^{k_{n}} \alpha_{n, j} \chi_{A_{n, j}}(x),
$$

where the sets $A_{n, 1}, \ldots, A_{n, k_{n}}$ are disjoint and $\cup_{j} A_{n, j}=B$. 
Using Hölder's and Young's inequalities we have

$$
\begin{aligned}
\int_{A_{n, j}} \frac{|f(y)|}{t} d \mu(y) & \leq\left(\int_{A_{n, j}}\left(\frac{|f(y)|}{t}\right)^{\alpha_{n, j}} d \mu(y)\right)^{1 / \alpha_{n, j}} \mu\left(A_{n, j}\right)^{1 / \alpha_{n, j}^{\prime}} \\
& \leq \frac{1}{\alpha_{n, j}} \int_{A_{n, j}}\left(\frac{|f(y)|}{t}\right)^{\alpha_{n, j}} d \mu(y)+\frac{\mu\left(A_{n, j}\right)}{\alpha_{n, j}^{\prime}} \\
& \leq \frac{1}{p_{B}^{-}} \int_{A_{n, j}}\left(\frac{|f(y)|}{t}\right)^{s_{n}(y)} d \mu(y)+\frac{\mu\left(A_{n, j}\right)}{\left(p_{B}^{+}\right)^{\prime}}
\end{aligned}
$$

Adding the inequalities from 1 to $k_{n}$ gives

$$
\int_{B} \frac{|f(y)|}{t} d \mu(y) \leq \frac{1}{p_{B}^{-}} \int_{B}\left(\frac{|f(y)|}{t}\right)^{s_{n}(y)} d \mu(y)+\frac{\mu(B)}{\left(p_{B}^{+}\right)^{\prime}} .
$$

This inequality holds for all $n$, hence the monotone convergence theorem implies that

$$
\mu(B)<\int_{B} \frac{|f(y)|}{t} d \mu(y) \leq \frac{1}{p_{B}^{-}} \int_{B}\left(\frac{|f(y)|}{t}\right)^{p(y)} d \mu(y)+\frac{\mu(B)}{\left(p_{B}^{+}\right)^{\prime}} .
$$

Since $p^{+}<\infty$, it follows that $\left(p_{B}^{+}\right)^{\prime}>1$, so we are done.

The following theorem is an adaptation of [2, Theorem 1.8]

5.2. Theorem. Let $X$ be a doubling space and assume that $p^{+}<\infty$. Then for all $f \in L^{p(\cdot)}(X)$ and $t>0$

$$
\mu(\{x \in X: \mathcal{M} f(x)>t\}) \leq C \int_{X}\left(\frac{|f(y)|}{t}\right)^{p(y)} d \mu(y) .
$$

Proof. Let $n>0$ and define

$$
\mathcal{M}_{n} f(x)=\sup _{r \leq n} f_{B(x, r)}|f(y)| d \mu(y) .
$$

Then the sequence $\left\{\mathcal{M}_{n} f(x)\right\}$ is increasing and converges to $\mathcal{M} f(x)$ for every $x \in X$. By the monotone convergence theorem, we find for each $t>0$ that

$$
\mu(\{x \in X: \mathcal{M} f(x)>t\})=\lim _{n \rightarrow \infty} \mu\left(\left\{x \in X: \mathcal{M}_{n} f(x)>t\right\}\right),
$$

and therefore it suffices to prove (5.3) for $\mathcal{M}_{n}$ and a constant independent of $n$.

Let $t>0$ and denote $E_{n}=\left\{x \in X: \mathcal{M}_{n} f(x)>t\right\}$. Then for each $x \in E_{n}$ there is a ball $B_{x}=B\left(x, r_{x}\right)$ such that

$$
f_{B_{x}}|f(y)| d \mu(y)>t .
$$

By the standard covering theorem [12, Theorem 1.2] we find a disjoint family $\mathcal{F}$ of balls $B_{x}$ with $E_{n} \subset \bigcup_{B \in \mathcal{F}} 5 B$. Using the doubling property and Lemma 5.1 this implies that

$$
\begin{aligned}
\mu\left(E_{n}\right) & \leq \sum_{B \in \mathcal{F}} \mu(5 B) \leq C \sum_{B \in \mathcal{F}} \mu(B) \\
& \leq C \sum_{B \in \mathcal{F}} \int_{B}\left(\frac{|f(y)|}{t}\right)^{p(y)} d \mu(y) \leq C \int_{X}\left(\frac{|f(y)|}{t}\right)^{p(y)} d \mu(y),
\end{aligned}
$$

where the constant $C$ depends on $p^{+}$and the doubling constant. 
A standard application of the weak type estimate is the Lebesgue point theorem. We show that this method also work in our case, provided we know that continuous functions are dense in $L^{p(\cdot)}(X)$. The theorem generalizes [11, Theorem 3.1] to metric measure spaces.

5.4. Theorem. Let $X$ be a locally compact doubling space and assume that $p^{+}<\infty$. Then for every $u \in L_{l o c}^{p(\cdot)}(X)$ and almost every $x \in X$

$$
\limsup _{r \rightarrow 0} f_{B(x, r)}|u(y)-u(x)|^{p(y)} d \mu(y)=0 .
$$

Proof. We may assume that $u \in L^{p(\cdot)}(X)$. Otherwise we can study a function $u \chi_{B_{r}}$, where $\chi_{B_{r}}$ is the characteristic function of a ball with radius $r>0$, and the theorem follows by the subadditivity of the measure. We define

$$
L u(x)=\limsup _{r \rightarrow 0} f_{B(x, r)}|u(y)-u(x)|^{p(y)} d \mu(y) .
$$

For $x \in X$ we obtain

$$
\begin{aligned}
L u(x) & \leq 2^{p^{+}} \limsup _{r \rightarrow 0} f_{B(x, r)}|u(y)|^{p(y)} d \mu(y)+2^{p^{+}} \limsup _{r \rightarrow 0} f_{B(x, r)}|u(x)|^{p(y)} d \mu(y) \\
& \leq 2^{p^{+}} \mathcal{M} u^{p(\cdot)}(x)+2^{p^{+}} \max \left\{|u(x)|,|u(x)|^{p^{+}}\right\}
\end{aligned}
$$

Furthermore $L\left(u_{1}+u_{2}\right)(x) \leq 2^{p^{+}} L u_{1}(x)+2^{p^{+}} L u_{2}(x)$ and if $v$ is continuous then $L v$ is identically zero. This yields

$$
\begin{aligned}
\mu(\{x \in X: L u(x)>t\}) \leq & \mu\left(\left\{x \in X: L(u-v)(x)>\frac{t}{2^{p^{+}}}\right\}\right) \\
\leq & \mu\left(\left\{x \in X: \mathcal{M}(u-v)^{p(\cdot)}(x)>\frac{t}{2^{p^{+}+2}}\right\}\right) \\
& +\mu\left(\left\{x \in X:|u(x)-v(x)|>\min \left\{\frac{t}{2^{p^{+}+2}}, \frac{t^{1 / p^{+}}}{2^{1+2}}\right\}\right\}\right) .
\end{aligned}
$$

We use the weak type estimate (4.1) or Theorem 5.2 for $(u-v)^{p(\cdot)} \in L^{1}(X)$ to get

$$
\begin{aligned}
\mu(\{x \in X: L u(x)>t\}) \leq & C(t) \int_{X}|u(y)-v(y)|^{p(y)} d \mu(y) \\
& +\mu(\{x \in X:|u(x)-v(x)|>C(t)\}) .
\end{aligned}
$$

Since continuous functions are dense in $L^{p(\cdot)}(X)$, Theorem 3.3, we can chose $u \rightarrow v$ in $L^{p(\cdot)}(X)$. Since convergence in norm implies convergence in measure, the righthand-side of the previous inequality tends to 0 for every $t>0$. By the subadditivity of the measure, this completes the proof.

\section{REFERENCES}

[1] E. Acerbi and G. Mingione: Regularity results for a class of functionals with non-standard growth, Arch. Ration. Mech. Anal. 156 (2001), 121-140.

[2] D. Cruz-Uribe, A. Fiorenze and C. J. Neugebauer: The maximal operator on variable $L^{p}$ spaces, Ann. Acad. Sci. Fenn. 28 (2003), 223-238.

[3] L. Diening: Maximal operator on generalized Lebesgue spaces $L^{p(\cdot)}$, Math. Inequal. Appl., to appear.

[4] L. Diening: Riesz Potential and Sobolev Embeddings of generalized Lebesgue and Sobolev Spaces $L^{p(\cdot)}$ and $W^{k, p(\cdot)}$, Math. Nachr., to appear.

[5] L. Diening and M. Růžička: Calderón-Zygmund operators on generalized Lebesgue spaces $L^{p(\cdot)}$ and problems related to fluid dynamics, J. Reine Ang. Math., to appear. 
[6] M. Eleutera: Hölder continuity results for a class of functionals with non standard growth, Boll. Unione Mat. Ital., to appear.

[7] X. Fan and D. Zhao: On the spaces $L^{p(x)}(\Omega)$ and $W^{m, p(x)}(\Omega)$, J. Math. Anal. Appl. 263 (2001), 424-446.

[8] P. Hajłasz and P. Koskela: Sobolev meets Poincaré, C. R. Acad. Sci. Paris 320 (1995), no. 1, 1211-1215.

[9] P. Hajłasz and P. Koskela: Sobolev met Poincaré, Mem. Amer. Math. Soc 145 (2000), no. 688.

[10] P. Harjulehto and P. Hästö: A capacity approach to the Poincaré inequality and Sobolev imbedding in variable exponent Sobolev space, Rev. Mat. Complut., to appear.

[11] P. Harjulehto and P. Hästö: Lebesgue points in variable exponent spaces, Reports of the Department of Mathematics, University of Helsinki, Preprint 364 (2003).

[12] J. Heinonen: Lectures on analysis on metric spaces, Springer-Verlag, New York, 2001.

[13] V. Kokilasvili and S. Samko: Maximal and fractional operators in weighted $L^{p(x)}$ spaces, Rev. Mat. Iberoamericana, to appear.

[14] O. Kováčik and J. Rákosník: On spaces $L^{p(x)}$ and $W^{1, p(x)}$, Czechoslovak Math. J. 41 (116) (1991), 592-618.

[15] J. Museliak: Orlicz Spaces and Modular Spaces, Springer-Verlag, Berlin, 1985.

[16] E. Nakai and H. Sumitomo: On generalized Riesz potentials and spaces of some smooth functions, Sci. Math. Jpn. 54 (2001), no. 3, 463-472.

[17] A. Nekvinda: Hardy-Littlewood maximal operator on $L^{p(x)}\left(\mathbb{R}^{n}\right)$, Math. Inequal. Appl., to appear.

[18] L. Pick and M. Růžička: An example of a space $L^{p(x)}$ on which the Hardy-Littlewood maximal operator is not bounded, Expo. Math. 19 (2001), 369-371.

[19] M. Růžička: Electrorheological Fluids: Modeling and Mathematical Theory, Springer-Verlag, Berlin, 2000.

[20] S. Samko: Convolution and potential type operators in $L^{p(x)}\left(\mathbb{R}^{n}\right)$, Integr. Transform. and Special Funct. 7 (1998), no. 3-4, 261-284.

[21] I. Sharapudinov: On the topology of the space $L^{p(t)}([0 ; 1])$, Matem. Zametki 26 (1979), no. 4, 613-632.

[22] E. Hewitt and K. Stromberg: Real and abstract analysis, Springer-Verlag, Berlin, 1965.

P. Harjulehto and M. Pere

Department of Mathematics

P.O. Box 4 (Yliopistonkatu 5)

FIN-00014 UNIVERSITY OF HELSINKI

FINLAND

P. Hästö

Department of Mathematics

UNIVERSITY OF MICHIGAN

Ann Arbor, MI 48109-1109

USA

Research group home-page:

http://www . math.helsinki.fi/analysis/varsobgroup

E-mail addresses:

petteri.harjulehto@helsinki.fi

peter.hasto@helsinki.fi

mikko.pere@helsinki.fi 